\title{
A human interstitial telomere associates in vivo with specific TRF2 and TIN2 proteins
}

\author{
Cécile Mignon-Ravix ${ }^{1}$, Danielle Depetris ${ }^{1}$, Bruno Delobel ${ }^{2}$, Marie-Françoise Croquette ${ }^{2}$ and \\ Marie-Geneviève Mattei*,1
}

\author{
${ }^{1}$ INSERM 491, Faculté de Médecine Timone, Marseille, France; ${ }^{2}$ Hopital Saint Antoine, Lille, France
}

\begin{abstract}
Mammalian telomeres are composed of long arrays of TTAGGG repeats that form a nucleoprotein complex which protects the chromosome ends. Human telomere function is known to require two TTAGGG repeat factors, TRF1 and TRF2, and several interacting proteins, but the mechanism by which the DNA/protein complex prevents end to end fusion in vivo has not been elucidated. In order to better understand the role of specific telomere-associated proteins in the organisation of chromosome ends, we have studied a patient with a rare chromosome rearrangement that has given rise to an interstitial telomere. Using specific antibodies and immuno-FISH on unfixed metaphase chromosomes, we show that the proteins TRF2 and TIN2 (TIN2 interacts with TRF1) co-localise with the interstitial TTAGGG repeats. Our results demonstrate, for the first time in humans, that TRF2 and TIN2 proteins associate with interstitial duplex TTAGGG repeats, in vivo. They confirm that double stranded-telomeric repeats, even when complexed with specific proteins, are not sufficient to create a functional telomere. Finally, they suggest a possible role for proteins in stabilising interstitial TTAGGG repeats.
\end{abstract}

European Journal of Human Genetics (2002) 10, 107 -112. DOI: 10.1038/sj/ejhg/5200775

Keywords: human interstitial telomere; TRF2; TIN2; proteins; immuno-FISH.

\section{Introduction}

Telomeres are specialised DNA/protein structures that act as protective caps to prevent end fusion and to distinguish the chromosome ends from double-stranded breaks. Mammalian telomeric DNA is composed of several kilobases of duplex TTAGGG repeats terminating in a $3^{\prime}$ single-stranded G-rich DNA tail. ${ }^{1}$

Telomeric DNA repeats have been shown to be associated with the specific TTAGGG repeat factors TRF1 and TRF2. Both factors bind in vitro to the duplex DNA repeats with high affinity $^{2}$ and binding does not require a DNA end., However, very low affinity binding to single-stranded DNA has recently been demonstrated, ${ }^{5}$ with a net preference for

*Correspondence: M-G Mattei, INSERM U491, Faculté de Médecine Timone, 27, Bd Jean Moulin, 13385 Marseille cedex, France.

Tel: +3304912571 73; Fax: +3304918043 19;

E-mail: genevieve.mattei@medecine.univ-mrs.fr

Received 10 October 2001; revised 23 November 2001; accepted 18 December 2001 the G-strand. TRF1 and TRF2 are found to co-localise, in vivo, with telomeres of human metaphase chromosomes. ${ }^{2,6,7}$ From a functional point of view, both have been suggested to stimulate formation of the terminal telomeric t-loop, with TRF1 coiling the duplex telomeric tract back on itself, allowing TRF2 to promote the invasion of the single-stranded $3^{\prime}$ overhang into the duplex telomeric repeat array. ${ }^{8}$ Moreover, TRF1 is thought to promote parallel pairing of telomeric DNA $^{2}$ and TRF2 to prevent chromosome fusion. ${ }^{9}$

Human telomeres contain other proteins that do not bind telomeric DNA directly and localize to telomeres via protein/ protein interaction, as is the case for TIN $2^{10}$ and tankyrase ${ }^{11}$ interacting with TRF1, and for hRap $1^{12}$ interacting with TRF2. Finally, although the $\mathrm{Ku}$ heterodimer is known to localise to telomeres via protein/protein interaction, it binds to DNA ends through its repair function, which renders possible its interaction with telomeric DNA. ${ }^{13-15}$

Nevertheless the mechanism by which the DNA/protein complex acts to prevent end-to-end fusion, in vivo, remains to be elucidated. 
In order to better understand the role of specific telomere-associated proteins in the organisation of chromosome ends, we tested for their presence at an interstitial telomere in a patient with a rearranged chromosome. This chromosome resulted from the translocation of an acentric chromosome 13 onto the short arm of an apparently intact $\mathrm{X}$ chromosome, creating a non functional interstitial telomere. In this study, using specific antibodies and immuno-FISH on unfixed metaphase chromosomes, we demonstrate, for the first time in humans, that the telomere-associated proteins TRF2 and TIN2 associate with interstitial TTAGGG repeats.

\section{Material and methods}

\section{Cytogenetic analysis of the patient}

The rearranged chromosome was fortuitously detected in a newborn referred for infectious disease, and presenting an isolated discrete lymphoedema of the feet, without any other evident clinical sign.

Routine chromosome analysis was performed on RHGbanded metaphase chromosomes obtained from PHA-stimulated peripheral blood lymphocytes, using standard protocols. In addition, the possibility that distal Xp sequences had been deleted from the rearranged chromosome was tested by fluorescence in situ hybridisation (FISH) with digoxigeninlabelled STS (steroid sulphatase) and KAL (Kalmann) cosmid probes (Oncor) and a biotin-labelled Xp/Yp-specific telomere probe (Clinisciences), all of which detect loci at the distal end of Xp.

Replication studies of the $\mathrm{X}$ chromosomes were performed using a very late terminal 5-bromo-2'-deoxyuridine (BrdU) pulse for the last $3 \mathrm{~h}$ of culture $(60 \mu \mathrm{g} / \mathrm{ml})$. BrdU incorporation was visualised using FITC-conjugated antiBrdU antibody (Boehringer-Mannheim) as previously described. ${ }^{16}$ The $\mathrm{X}$ chromosomes were identified by an $\mathrm{X}$ alphoid digoxigenin-labelled probe (Oncor), hybridised in situ to the preparations before the immunostaining protocol.

In order to search for telomeric TTAGGG repeats, a FITClabelled PNA (CCCTAA) 3 probe (DAKO) was hybridised in situ to fixed metaphase chromosomes, as recommended by the supplier.

All preparations subjected to FISH were then counterstained with antifade (Vectashield, Vector Laboratories) containing $100 \mathrm{ng} / \mathrm{ml}$ DAPI (4,6-diamidino 2-phenyl indole) and analysed by fluorescence microscopy.

\section{Specific antibodies}

The presence of two telomere-specific proteins at the interstitial telomere was studied: the TTAGGG repeat factor TRF2 and the TRF1-interacting protein TIN2. TRF2 was detected with a rabbit polyclonal antibody raised against aminoacids AA306 to AA391 (kind gift from E Gilson and K Ancelin, ENS Lyon, France). TIN2 was detected using a rabbit polyclonal antibody raised against AA121 to AA354, ${ }^{10}$ kindly provided by J Campisi and $\mathrm{SH}$ Kim (Berkeley, USA).

\section{Immuno-FISH protocol}

Unfixed chromosome preparations were obtained from an EBV-transformed lymphoblastoid cell line derived from the patient. After colchicin treatment, cells were incubated in hypotonic solution (diluted foetal bovine serum, 20\%) for $10 \mathrm{~min}$ at $37^{\circ} \mathrm{C}$. They were then collected by centrifugation and resuspended in fresh hypotonic solution, the cell density being adjusted to $10^{4}-10^{5}$ cells $/ \mathrm{ml}$. Cells were kept on ice and metaphase spreads were prepared within $1 \mathrm{~h}$. Aliquots $(0.5 \mathrm{ml})$ of cell suspension were centrifuged $(300 \mathrm{t} /$ min for $10 \mathrm{~min}$ ) onto polylysine-coated slides in a cytospin 3 (Shandon). For the experiment with TIN2, the quality of spread preparations was improved by adding glycerol $(0.5 \%)$ to the cell suspension before centrifugation. The metaphase preparations were then fixed at room temperature for $10 \mathrm{~min}$ in freshly prepared $4 \%(\mathrm{w} / \mathrm{v})$ paraformaldehyde/PBS pH 7.2. Slides were then rinsed briefly in PBS and used immediately.

A permeabilisation step was performed with $0.1 \%$ Triton $\mathrm{X}$ 100 in PBS for $40 \mathrm{~min}$ at room temperature, followed by at least 15 min in blocking buffer (PBS/0.05\% Triton X-100/5\% goat serum $/ 5 \%$ fotal calf serum). Cell preparations were then incubated with buffer (PBS/0.05\% Triton X-100) containing either anti-TRF2 diluted 1/1000, or anti-TIN2 diluted 1/50, for $2 \mathrm{~h}$ at room temperature. DNAse $1(10 \mathrm{ng} / \mathrm{ml})$ was added to the primary incubation mixture in order to improve antibody accessibility. ${ }^{7}$ After washing $(3 \times 5 \mathrm{~min})$ in PBS, primary antibodies were detected with a Cyanin 3-conjugated affinity purified goat anti-rabbit IgG (Tebu) diluted $1 / 300$ in PBS/0.05\% Triton X-100, for $1 \mathrm{~h}$ at room temperature. Preparations were then fixed with $4 \%(\mathrm{w} / \mathrm{v})$ paraformaldehyde in PBS for 5 min and counterstained with DAPI (100 $\mathrm{ng} / \mathrm{ml})$ diluted in vectashield.

All antibodies were tested in individual staining reactions for their specificity and performance. Controls without primary antibodies were all negative. To exclude potential artifacts induced by FISH, the immunofluorescent signal given by each antibody on metaphase chromosomes was first analysed and photographed. The same preparations were subsequently subjected to the double colour FISH protocol using both a chromosome 13 digoxigenin-labelled paint probe and a chromosome $\mathrm{X}$ biotin-labelled paint probe (Oncor) and the signals detected as recommended by the manufacturer. Preparations were then counterstained with DAPI at $100 \mathrm{ng} / \mathrm{ml}$ in vectashield and re-photographed.

\section{Microscopy analysis}

Metaphase preparations were all observed using an Axioplan2 Zeiss fluorescent microscope. The images were captured with a CCD camera (photometrics 'Sensys'). Information was collected and merged using IPLabs Spectrum software (Vysis). 


\section{Results}

Characterisation of the karyotype of the patient

Standard banding techniques showed that the karyotype of the patient consisted of only 45 chromosomes, with an acentric chromosome 13 translocated onto the short arm of an apparently normal $\mathrm{X}$ chromosome. The formula was $45, \mathrm{X},-13,-\mathrm{X},+\operatorname{der} \mathrm{X} \mathrm{t}(\mathrm{X} ; 13)(\mathrm{p} 22 ; \mathrm{q} 11)$. We detected no loss of $\mathrm{X}$ chromosome material on the rearranged der $\mathrm{X}$, using either RHG-banding technique or FISH with various distal Xp telomeric probes (STS, KAL, and the Xp/Yp specific telomere)(data not shown).

Moreover, FISH with a specific FITC-labelled PNA telomere probe allowed us to identify interstitial, non-functional telomeric TTAGGG repeats on the derived chromosome, located close to the translocation junction (Figure 1). These interstitial telomeric repeats are presumably derived from the $\mathrm{Xp}$ telomere. In all metaphases analysed, the FISH-signal obtained at the interstitial site appeared very similar in intensity to the signals located at the extremities of the rearranged chromosome. Despite the presence of internal telomeric sequences, the rearranged chromosome appeared to be stable in 200 metaphases analysed.

Inactivation studies demonstrated that the translocated $\mathrm{X}$ chromosome was inactivated in 49/60 (81.6\%) metaphases with detectable BrdU incorporation. However, no difference in BrdU incorporation was detected between the normal and

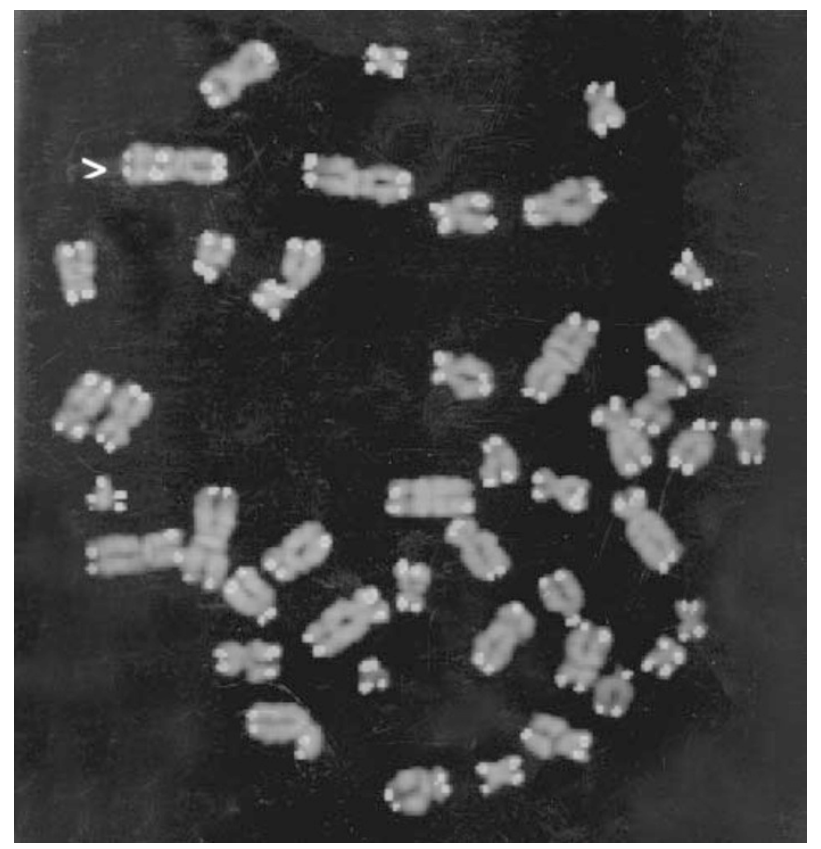

Figure 1 The TTAGGG repeats are detected by fluorescence in situ hybridisation on fixed metaphase chromosomes. FITClabelled PNA (CCCTAA) ${ }_{3}$ probe hybridised on metaphase of the patient shows fluorescent spots on the telomere ends of each chromosome and an interstitial signal on the rearranged chromosome. the translocated chromosome 13 (data not shown), suggesting that inactivation had not spread to the translocated autosomal segment. This could result from the presence of interstitial repetitive DNA sequences, as was previously observed for a similar rearrangement involving chromosome 9 beta-satellite sequences. ${ }^{17}$ It might also be the result of a limiting amount of Xi specific transcript (XIST) synthesised from the XIST gene on the inactive X chromosome. For the translocation product described here, XIST transcript level would then only be sufficient to inactivate the entire $\mathrm{X}$ chromosome, but not the adjacent chromosome 13 .

\section{Immunostaining results}

Unfixed metaphases of the patient were subjected to immunostaining with two telomere-specific proteins, TRF2 and TIN2 (which interacts with TRF1). The rearranged chromosome was subsequently identified by double colour FISH performed on the same preparations.

Immunofluorescence performed with the anti-TRF2 polyclonal antibody revealed a punctate pattern in interphase nuclei and one or two spots at the ends of metaphase chromosomes. Moreover, on well spread metaphases, a clear interstitial protein signal was detected on a large chromosome. This chromosome was then identified as the translocation product by FISH, and the anti-TRF2 signal shown to be located at the junction between chromosome 13 and the chromosome X short arm (Figure 2).

In order to improve protein preservation during the spreading protocol, unfixed metaphase preparations were obtained with the addition of $0.5 \%$ glycerol to the cell suspension before the cytospin step. Under these conditions, the anti-TIN2 antibody resulted in a signal distribution on metaphase chromosome that was very similar to that obtained with anti-TRF2 antibody. In several metaphases, where the rearranged chromosome lay at the periphery, a distinct signal was visible at the interstitial telomere, demonstrating the presence of TIN2 protein and suggesting the presence of TRF1 protein at this site (Figure 3). ${ }^{10}$

\section{Discussion and conclusion}

In order to better understand the role of specific telomereassociated proteins in telomere organisation, we tested for their presence at an interstitial and non functional telomere in a patient with an $(\mathrm{X} ; 13)$ translocation. Using specific antibodies and immuno-FISH on unfixed metaphase chromosomes, we showed the co-localisation of telomeric DNA and the two telomeric proteins TRF2 and TIN2 at the translocation breakpoint.

We first showed that TRF2 associates with interstitial duplex TTAGGG repeats. TRF2 has been demonstrated to specifically bind duplex telomeric DNA sequences in vitro and to localize in vivo at the telomeres of interphase and metaphase chromosomes. ${ }^{4,6,7}$ Nevertheless, electron microscopy studies have raised doubts as to whether TRF2 binds to 


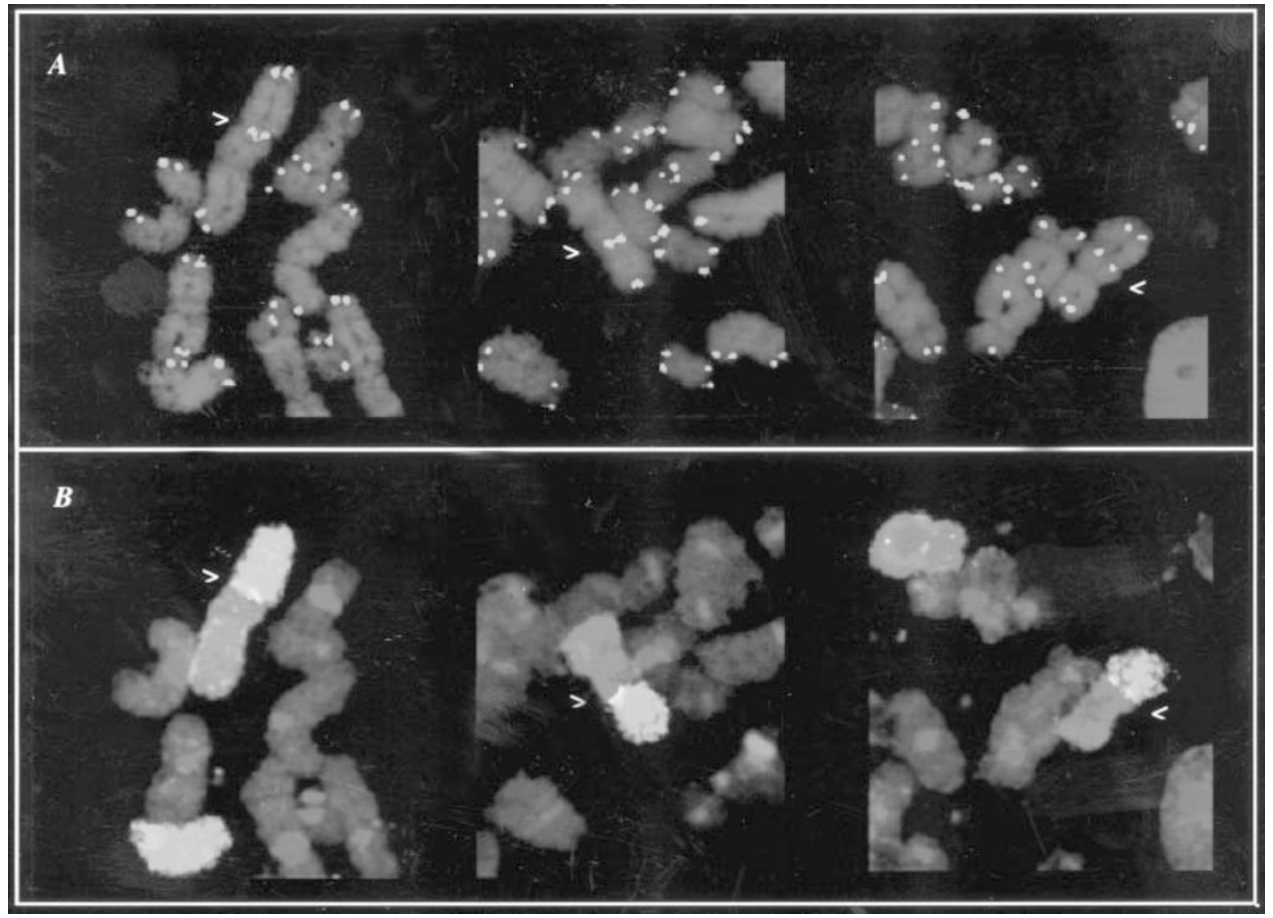

Figure 2 The TRF2 protein is present at the interstitial telomere of the rearranged chromosome (indicated by an arrowhead). (A) Three partial unfixed metaphases after immunostaining with anti-TRF2 specific polyclonal antibody. (B) The rearranged chromosome is subsequently identified by FISH using chromosome 13 and chromosome $\mathrm{X}$ paint probes.

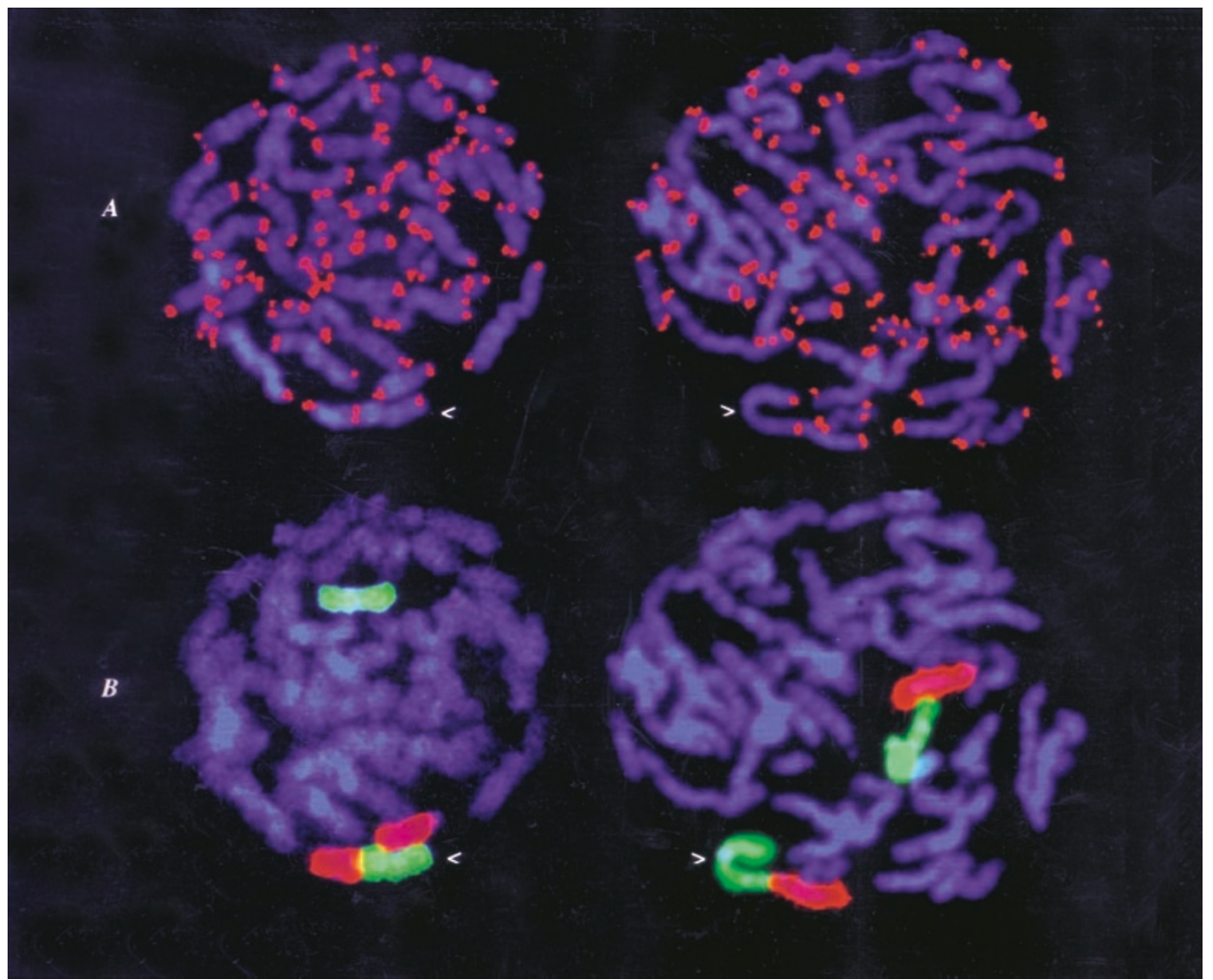

Figure 3 The TIN2 protein is present at the interstitial telomere of the rearranged chromosome (indicated by an arrowhead). (A) Two unfixed metaphases after immunostaining with anti-TIN2 specific polyclonal antibody. (B) The arranged chromosome is subsequently identified by FISH using chromosome 13 (red) and chromosome $\mathrm{X}$ (green) paint probes. 
duplex telomeric DNA or to a particular structural feature of telomeres in vivo. ${ }^{8,18}$ In the rearranged chromosome described here, the internal repeats do not appear to be organised as a functional telomere, and no features evocating a telomeric structure are observed. Moreover, it is reasonable to assume that no single-stranded DNA is present within the interstitial repeats, since if left unrepaired it would lead to broken chromatids detectable in metaphases. We observed no such instability in metaphases, and our results are therefore consistent with the hypothesis that TRF2 binds directly to human duplex telomeric DNA in vivo.

We next showed that TIN2 associates with interstitial TTAGGG repeats in metaphases. Nevertheless, TIN2 does not bind double-stranded TTAGGG repeats directly, and has been demonstrated to localise to telomeres through its interaction with TRF1. ${ }^{10}$ Although we cannot exclude the intervention of other factors, our results suggest that TRF1 is present at the interstitial TTAGGG repeats. In agreement with this hypothesis, TRF1 has been demonstrated to colocalise with large interstitial TTAGGG repeats in immortal Chinese Hamster ovary cells. $^{19}$

Our results therefore confirm that double-stranded telomeric repeats, even when complexed with specific telomere proteins which normally prevent chromosome fusion, ${ }^{9}$ are not sufficient to create a functional telomere. In functional telomeres, a 3' single-stranded G-rich DNA tail has been demonstrated to form a t-loop in vitro, in the presence of both the TTAGGG double-stranded DNA, and the TRF2 protein. ${ }^{8}$ On the basis of the lack of single-stranded DNA and the presence of double-stranded DNA and TRF2 at the site of interstitial telomere, our results highlight the role of the 3' single-stranded G-rich DNA tail in the organisation of a functional telomere. We suggest that it is the lack of a single-stranded DNA tail at the internal repeats that prevents the formation of a t-loop structure even in the presence of TRF2.

The internal telomeric TTAGGG repeat appears to be stable. Interstitial telomeric sequences are rarely found in human constitutional pathologies ${ }^{20-26}$ although they are more commonly reported in tumoral pathologies. ${ }^{27-29}$ In mammals, internal telomeric sequences have been shown to be hotspots for meiotic recombination. ${ }^{30,31}$ Moreover, they are mitotically unstable being the site of breakage, fragility and jumping translocations which result from somatic recombination between the interstitial telomere and the telomeres of normal chromosomes. ${ }^{32-34}$ It has also been shown that transfection of plasmids containing telomere repeat sequences results in chromosome breaks. ${ }^{35,36}$ Strikingly, the interstitial repeats described here show great stability.

This stability could be the result of a very low copy number of the repeat sequence at the internal telomere, which might decrease the probability of somatic recombination events. However, the intensity of the FISH signal (by direct microscopic observation under non-saturated conditions) did not appear significantly different at the internal telomere compared to the normal telomeres.

The stability of internal TTAGGG repeats, in this case, might also be linked to the late replication of the translocated $(\mathrm{X} ; 13)$ chromosome. As a result, the translocated chromosome could be isolated in an heterochromatic structure, that allows it to escape non homologous somatic recombination.

Finally, the stability of these internal repeats may also be conferred by the presence of specific telomere proteins, such as TRF2, TIN2 and possibly TRF1. TRF2 and TRF1 might recognise the interstitial TTAGGG repeats, with other proteins, such as TIN2, promoting a compact stable structure. In support to this idea TRF1 and TRF2, which bind to the stable interstitial TTAGGG blocks in immortal Chinese Hamster ovary cells, ${ }^{18,19}$ have been suggested to promote stability by reducing unequal homologous recombination between TTAGGG sequences. ${ }^{19}$

In conclusion, the immuno-localisation of telomereassociated proteins in different pathological situations should help us to understand the organisation of the DNA/ protein complex found at internal and distal telomere sites of chromosomes, and the mechanisms involved in telomere stabilisation, in vivo.

\section{Acknowledgments}

The authors thank Drs E Gilson and K Ancelin (ENS, Lyon, France), Drs J Campisi and SH Kim (LBNL, Berkeley, USA) who kindly provided the antibodies. They also thank Dr E Gilson for very helpful discussion and Dr M Mitchell for reviewing the English text. They also thank the family for its participation. This work was supported by INSERM, the Association pour la Recherche contre le Cancer (ARC), and the ACI 2000 from the ministere de la Recherche.

\section{References}

1 Makarov V, Hirose Y, Langmore JP: Long G tails at both ends of human chromosomes suggest a $C$ strand degradation mechanism for telomere shortening. Cell 1997; 88: 657-666.

2 Griffith J, Bianchi A, De Lange T: TRF1 promotes parallel pairing of telomeric tracts in vitro. J Mol Biol 1998; 278: 79-88.

3 Zhong Z, Shiue L, Kaplan S, De Lange T: A mammalian factor that binds telomeric TTAGGG repeats in vitro. Mol Cell Biol 1992; 12: $4834-4843$.

4 Broccoli D, Smogorzewska A, Chong L, De Lange T: Human telomeres contain two distinct Myb-related proteins, TRF1 and TRF2. Nat Genet 1997; 17: 231 - 235.

5 Vassetzky NS, Gaden F, Brun C, Gasser SM, Gilson E: Taz1p and Teb1p, two telobox proteins in Schizosaccharomyces pombe, recognise different telomere-related DNA sequences. Nucleic Acids Res 1999; 27: 4687-4694.

6 Chong L, Van Steensel B, Broccoli D, Erdjument-Bromage H, Hanish J, Tempst PC: A human telomeric protein. Science 1995; 270: $1663-1667$

7 Bilaud T, Brun C, Ancelin K, Koerig CE, Laroche T, Gilson E: Telomeric localization of TRF2, a novel human telobox protein. Nat Genet 1997; 17: 236-239.

8 Griffith J, Comeau L, Rosenfield S et al. Mammalian telomeres end in a large duplex loop. Cell 1999; 97: 503-514.

9 van Steensel B, Smogorzewska A, De Lange T: TRF2 protects human telomeres from end-to-end fusions. Cell 1998; 92: $401-$ 413. 
10 Kim SH, Kaminker P, Campisi J: TIN2, a new regulator of telomere length in human cells. Nat Genet 1999; 23: 405-412.

11 Smith S, Giriat I, Schmitt A, De Lange T: Tankyrase, a poly(ADPribose) polymerase at human telomeres. Science 1998; 282: $1484-1487$.

$12 \mathrm{Li} \mathrm{B}$, Oestreich S, de Lange T: Identification of human Rap1: implications for telomere evolution. Cell 2000; 101: $471-483$.

13 Bianchi A, de Lange T: Ku binds telomeric DNA in vitro. J Biol Chem 1999; 274: 21223-21227.

14 Hsu HL, Gilley D, Galande SA et al. Ku acts in a unique way at the mammalian telomere to prevent end joining. Genes Dev 2000; 15: $2807-2812$.

15 d'Adda di Fagagna F, Hande MP, Tong W et al: Effects of DNA nonhomologous end-joining factors on telomere length and chromosomal stability in mammalian cells. Curr Biol 2001; 11: $1192-1196$

16 Stavropoulou C, Mignon C, Delobel B et al: Severe phenotype resulting from an active ring $\mathrm{X}$ chromosome in a female with a complex karyotype: characterisation and replication study. $J$ Med Genet 1998; 35: 932 -938.

17 Mattei MG, Mattei JF, Ayme S, Malpuech G, Giraud F: A dynamic study in two new cases of $\mathrm{X}$ chromosome translocations. Hum Genet 1978; 41: $251-257$.

18 Smogorzewska A, Van Steensel B, Bianchi A et al: Control of human telomere length by TRF1 and TRF2. Mol Cell Biol 2000; 20: $1659-1668$.

19 Krutilina RI, Oei SL, Buchlow G et al: A negative regulator telomere-length protein TRF1 is associated with interstitial (TTAGGG)n blocks in immortal chinese hamster ovary cells. Biochem Biophys Res Commun 2001; 280: 471-475.

20 Petit C, Levilliers J, Weissenbach J: Physical mapping of the human pseudoautosomal region: comparison with genetic linkage map. EMBO J 1988; 7: 2369-2376.

21 Jedele KB, Michels VV, Thibodear SN et al: Demonstration of telomeric DNA at the fusion site in a constitutional dicentric X;22 chromosome translocation. Am J Hum Genet 1989; 45(suppl): A102.

22 Park VM, Gustashaw M, Wathen TM: The presence of interstitial telomeric sequences in constitutional chromosome abnormalities. Am J Hum Genet 1992; 50: 914-923.

23 Devriendt K, Petit P, Matthijs G et al: Trisomy 15 rescue with jumping translocation of distal $15 \mathrm{q}$ in Prader-Willi syndrome. $J$ Med Genet 1997; 34: 395 - 399.
24 Vermeesch JR, Petit P, Speleman F, Devriendt K, Fryns JP, Marynen P: Interstitial telomeric sequences at the junction site of a jumping translocation. Hum Genet 1997; 99: 735 - 737.

25 Jewett T, Marnane D, Stewart W et al: Jumping translocation with partial duplications and triplications of chromosomes 7 and 15. Clin Genet 1998; 53: $415-420$.

26 Reddy KS, Murphy T: Fusion of 9 beta-satellite and telomere (TTAGGG)n sequences results in a jumping translocation. Hum Genet 2000; 107: 268-275.

27 Cuthbert G, McCullough S, Finney R, Breese G, Bown N: Jumping translocation at 11q23 with MLL gene rearrangement and interstitial telomeric sequences. Genes Chromosomes Cancer 1999; 24: $295-298$.

28 Busson Le Coniat M, Brizard F, Smadja NV, Maarek O, Der Sarkissian H, Berger R: Interstitial telomere repeats in translocations of hematopoietic disorders. Leukemia 2000; 14: 1630 1633.

29 Fan YS, Rizkalla K, William BF, Engel CJ: Jumping translocations of $11 \mathrm{q}$ in acute myeloid leukemia and $1 \mathrm{q}$ in follicular lymphoma. Cancer Genet Cytogenet 2000; 118: $35-41$.

30 Ashley T, Ward DC: A 'hot spot' of recombination coincides with an interstitial telomeric sequence in the Armenian hamster. Cytogenet Cell Genet 1993; 62: 169-171.

31 Mondello C, Pirzio L, Azzalin CM, Giulotto E: Instability of interstitial telomeric sequences in the human genome. Genomics 2000; 68: $111-117$.

32 Drets ME, Therman E: Human telomeric 6; 19 translocation chromosome with a tendency to break at the fusion point. Chromosoma 1983; 88: 139-144.

33 Hastie ND, Allshire RC: Human telomeres: fusion and interstitial sites. Trends Genet 1989; 5: 326-331.

34 Ancelin K, Brun C, Gilson E: Role of the telomeric DNA-binding protein TRF2 in the stability of human chromosome ends. Bioessays 1998; 20: 879-883.

35 Farr C, Fantes J, Goodfellow P, Cooke H: Functional reintroduction of human telomeres into mammalian cells. Proc Natl Acad Sci USA 1991; 88: 7006-7010.

36 Murnane JP, Yu LC: Acquisition of telomere repeat sequences by transfected DNA integrated at the site of a chromosome break. Mol Cell Biol 1993; 13: 977 - 983. 